\title{
Accessibility to Facilities for Persons with Disabilities at Public Institutes of Higher Learning
}

\author{
Aizan Sofia Amin, Siti Zuliana Md Zuki, Noremy Md Akhir
}

\begin{abstract}
Issues related to Persons with Disabilities $(P W D)$ rights are increasingly being considered in Malaysia. This includes their rights in education, employment, healthcare as well as access to the facilities and services provided. Accessibility in education especially at universities are among the major issues faced by PWD. Therefore, this study was conducted to identify the accessibility of facilities for persons with disabilities in public institutes of higher education. This study focuses on structured observations of PWD facilities at four faculties and four resource centres in Universiti Kebangsaan Malaysia. Five basic facilities for PWD such as parking space, stairs, lifts, toilets and pathways/ramps were thoroughly observed. A detailed comparison was carried out to identify the accessibility of those facilities and the extent of compliance to specifications outlined in universal design criteria. The study findings show that although PWD facilities were available, those facilities were still inadequate and did not follow the specifications set. Facility providers namely the university should devise a specific action plan and establish an inclusive policy for PWD to ensure their rights and needs are entirely fulfilled.
\end{abstract}

Index Terms: Accessibility, Facilities, Persons With Disabilities, Universal Design

\section{INTRODUCTION}

The Minister of Education's 2019 Mandate titled 'Education for All, Responsibility for All', stated that education is the true path to achieving Vision 2020. In efforts to create a democratic, morally upright and ethical society, as well as being capable in various aspects, no one should be neglected. This means that all individuals have the right to receive quality education regardless of their background, social status or physical ability. This is supported by Bodaghi and Zainab [1] who claim that access to all facilities,

Revised Manuscript Received on September 25, 2019

Aizan Sofia Amin, Research Centre for Psychology and Human Wellbeing, Faculty of Social Sciences and Humanities, Universiti Kebangsaan Malaysia, Malaysia.

Siti Zuliana Md Zuki, Research Centre for Psychology and Human Wellbeing, Faculty of Social Sciences and Humanities, Universiti Kebangsaan Malaysia, Malaysia.

Noremy Md Akhir, Research Centre for Psychology and Human Wellbeing, Faculty of Social Sciences and Humanities, Universiti Kebangsaan Malaysia, Malaysia. resources and services is the right of every individual in society despite differences in abilities and limitations. Statistics released by the Department of Statistics Malaysia on November 28, 2018 show that there were 453258 PWD registered with the Department of Social Welfare in 2017.

The increasing number of PWD directly affects the increasing number of disabled students in institutions of higher education. This shows that PWD are just as capable in education as other individuals. However for disabled students, obstacles begin to arise due to external factors such as their surroundings that are not disabled friendly. To produce successful disabled graduates, two key barriers need to be overcome. Firstly, specialized services must be provided to maximise the ability of students to fully participate in their chosen course of study. Secondly, in terms of facilities on campus. The campus must have a disabled friendly design in addition to basic facilities that can be easily accessed by PWD [2].

The Persons with Disability Act 2008 (Act 685) also provides for facilities for the disabled in Article 26:

"Persons with disabilities shall have the right to access to and use of, public facilities, amenities, services and buildings open or provided to the public on equal basis with persons without disabilities, but subject to the existence or emergence of such situations that may endanger the safety of persons with disabilities."

However, a study by Hazlin and Safura [3] at an institute of higher learning in Klang Valley found that disabled facilities were still not fully provided by management. Basic facilities that were provided such as pathways/ramps, rails, lifts and stairs were not comprehensive and caused barriers to the movements of the disabled. Meanwhile, basic facilities at Universiti Kebangsaan Malaysia were also at a minimum particularly at student focal areas such as student association buildings, lecture halls, residential colleges and sports complexes [4]. As such, this study is carried out to identify the facilities provided at Universiti Kebangsaan Malaysia (UKM), Bangi Campus according to areas that are the main focus of students. In addition, this study also identified whether the facilities provided complied with Universal Design Guidelines issued by the government. Blue Eyes Intelligence Engineering 


\section{LITERATURE STUDY}

\section{A. Acts and laws}

In year 1994, Standard Rules of Equal Opportunity for Persons with Disability was introduced based on advocacy from United Nations Human Rights Council (UNHRC). Consequently, in 2006, a convention regarding PWD rights, namely UNCRPD (United Nations Convention on the Rights of People with Disabilities) was organized to empower the rights of the disabled to ensure social and development equality in all aspects of life. One of the essence of this convention is that all participating countries must identify and eliminate accessibility obstructions and constraints to buildings and physical surroundings.

Among the major challenges often faced by PWD in Malaysia are related to issues of transportation and physical environments [5]. Like other developing countries, Malaysia has also taken proactive steps by providing disabled facilities to address these constraints. Apart from PWD Act 2008 (Act 685), the government under the Ministry of Women, Family and Community Development drafted a PWD Action Plan 2016-2022 blueprint. There are 10 strategic cores emphasized in the action plan. For the purpose of this study, Strategic Core 1 that is to increase PWD accessibility is seen as the most significant. One of the main objectives of the strategic core is to increase the provision of access to physical surroundings in both urban and rural settings based on universal design concepts. Various ministries and government agencies are involved in the planning of short-term plans (2016-2018) and long-term plans (2016-2022).

Taking a look at governmental departments, access to disabled facilities have improved but is not fully implemented. The Ministry of Housing and Local Government has also issued guidelines for universal design (2015) which outlines the need to plan more facilities and urban settings that feature universal design. These facilities must be accessible to all individuals including PWD without any physical obstructions. For Malaysia and other developing countries, the awareness and inclusion of universal design is still at its infancy. Although there are commendable acts, laws and action plans on this issue, the enforcement authorities involved do not strictly enforce the need to provide disabled access in each surrounding [6].

\section{B. PWD facilities in Malaysia}

The concept of universal design was introduced by a disabled architect named Ronald Mace in year 1985. He defined universal design as the concept of designing all products and the built environment to be aesthetic and usable to the greatest extent possible by everyone, without the need for adaptation or specialized design regardless of their age, ability, or status in life [7]. Apart from being used in the field of architecture, this concept is also accepted in the field of engineering as well product and landscape design. Another issue that needs to be addressed other than the design of the amenities is in terms of accessibility. Accessibility is defined as design quality of environments that can be accessed by people with disabilities and senior citizens. It is closely related to the barrier-free design concept that eliminates all structural obstacles that exist in a particular building [8][9].

The study carried out by Syazwani and Mariam [10] identified basic amenities that are a key neccesity to the disabled. For the visually impaired, they need tactile blocks, warning signs for indoor facilities and building signage that can provide them with information of their location. On the other hand, for the physically disabled, a pathway or ramp is crucially needed as they face difficulties navigating areas with steep gradients. Apart from that, they also face obstacles at ablution areas as well as toilets that are not disabled friendly in terms of utensils, size and layout.

\section{PWD facilities at institutes of higher learning}

Internationally, efforts to assist PWD in accessibility issues are actively carried out. Universities in the United Kingdom for example are required to respect and ensure that PWD rights are complied with under the Equality Act 2010 and United Nations Convention. For example, at Oxford University they take great care in assisting with the travelling and movements of the disabled on campus. It can be observed from the university's website that access guides are provided in each department which makes it easier for PWD to plan their movements prior to arriving at their destination. This also facilitates PWD in assessing the extent of disabled facilities provided at the university before applying for admission there.

Public institutes of higher learning in Malaysia are also increasingly concerned about PWD accessibility issues. For example, Universiti Malaya (UM) and Universiti Islam Antarabangsa Malaysia (UIAM). Both universities have established PWD inclusive policies aimed at fulfilling and protecting the rights of disabled staff and students whilst on campus. In correspondence to the increase of PWD at both universities, disabled facilities are also being added at most institutions of higher learning. However, easily accessible facilities are still lacking at the main buildings on campus. Most of the buildings have disabled facilities, but these facilities do not comply with the specifications set for disabled facilities. As a result, these conditions make it difficult for PWD to access these facilities.

There are five major barriers that are often faced by disabled students at institutes of higher learning namely access to higher education, access to information, physical surroundings, assumptions of 'normality' and level of disability awareness [11]. In this study, the researcher only focused on facilities as it is deemed to significantly impact disabled students at universities. This is because quality facilities will facilitate the movements of the students thus enabling them to live life as the other students. A study by Tinklin and Hall [11] also found that some disabled students were unable to further their studies in their chosen course despite being eligible due to the unavailability of facilities for the physically disabled at the faculty.

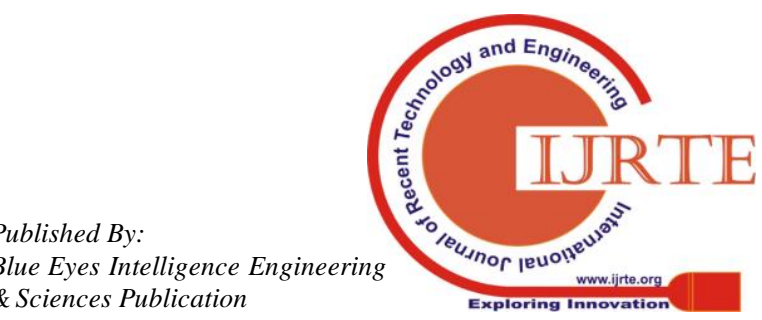


This matter is extremely disappointing as the PWD right to receive equal education as other students is neglected.

In order to enable PWD to receive quality education, comprehensive facilities catered to their needs should be provided. In Malaysia, studies regarding disabled facilities are increasingly being conducted. However, there are still gaps that need to be filled particularly on the provision of basic facilities. For example, a study conducted on visually impaired students at University Malaya found that pedestrian areas, toilets, recreational areas, transportation, libraries, residential halls and lecture halls were areas that were in dire need of specialized disabled facilities. Furthermore, among the facilities that should be given more attention are the pedestrian areas and the toilets. Findings from the study suggests that these facilities should be added to areas that become the focal area for the disabled [12]. Even though facilities for the disabled were provided, there still exists many shortcomings including facilities that were inaccessible, inadequate and of poor quality [13]. This includes issues of facilities that could not be accessed due to being too far as well as facilities that that did not comply with the specifications set for PWD facilities. Therefore, this study was conducted to identify accessibility of PWD facilities at Universiti Kebangsaan Malaysia as one of the nation's public institute of higher learning.

\section{METHODOLOGY}

This study uses a qualitative approach that is by case studies. Case studies were conducted to collect data, obtain meaning and gain insight from a case [14]. In this study, the researchers used case studies to observe and identify the types of facilities provided for physically disabled and visually impaired individuals at four faculties and four student resource centres in UKM. All four faculties were chosen based on the higher number of disabled students there compared to other faculties.

\begin{tabular}{|l|c|}
\hline FACULTY/ CENTRE & $\begin{array}{l}\text { NUMBER OF PWD } \\
\text { STUDENTS }\end{array}$ \\
\hline Faculty of Education (FPend) & 11 \\
\hline $\begin{array}{l}\text { Faculty of Social Sciences } \\
\text { and Humanities (FSSK) }\end{array}$ & 10 \\
\hline $\begin{array}{l}\text { Faculty of Science and } \\
\text { Technology (FST) }\end{array}$ & 7 \\
\hline $\begin{array}{l}\text { Faculty of Economics and } \\
\text { Management (FEP) }\end{array}$ & 6 \\
\hline
\end{tabular}

The four resource centres selected are Tun Seri Lanang Library (PTSL), Pusanika, Wisma UNIKEB and Academic Management Centre (BPA). Based on observations made, these four places are the most frequented by students in UKM. The researchers then compared all the facilities provided at all the selected places against the Universal Design Guidelines issued by the Department of Local
Government, Ministry of Urban Wellbeing, Housing and Local Government 2015.

Five basic facilities needed by PWD were selected for detailed observation in this study namely parking space, stairs, lifts, toilets and pathways /ramps. Previous studies that were conducted on disabled facilities at several hospitals also listed these five facilities as key facilities needed by PWD in daily life [15]. This study also focused on facilities that were used the most by the physically disabled and visually impaired as the number of PWD from both categories are the highest at UKM.

The results from this study will compare the facility characteristics at several building in UKM with the characteristics that are specified in the universal design criteria. From the results of the study, the parties involved will be able to identify the extent of improvements needed to facilitate the disabled using the facilities. 


\section{STUDY RESULTS}

A. PARKING SPACE

\begin{tabular}{|c|c|c|c|c|c|c|c|c|}
\hline $\begin{array}{l}\text { Universal } \\
\text { design indoor } \\
\text { criteria }\end{array}$ & $\begin{array}{l}\text { Perpustakaan } \\
\text { Tun Seri } \\
\text { Lanang } \\
\text { (PTSL) } \\
\end{array}$ & PUSANIKA & UNIKEB & $\begin{array}{c}\text { ACADEMIC } \\
\text { MANAGEMENT } \\
\text { SECTION } \\
\text { (BPA) } \\
\end{array}$ & $\begin{array}{c}\text { FACULTY OF } \\
\text { SOCIAL SCIENCES } \\
\text { AND HUMANITIES }\end{array}$ & $\begin{array}{l}\text { FACULTY OF } \\
\text { EDUCATION }\end{array}$ & $\begin{array}{c}\text { FACULTY OF } \\
\text { ECONOMICS } \\
\text { AND } \\
\text { MANAGEMENT }\end{array}$ & $\begin{array}{c}\text { FACULTY OF } \\
\text { SCIENCE AND } \\
\text { TECHNOLOG } \\
\text { Y } \\
\end{array}$ \\
\hline $\begin{array}{l}\text { Number of } \\
\text { disabled } \\
\text { parking space } \\
\end{array}$ & Only one & Only two & Only one & Not provided & Only three & Only one & Only one & Only one \\
\hline $\begin{array}{l}\text { Wheelchair } \\
\text { user parking } \\
\text { symbol on } \\
\text { parking space } \\
\text { surface }\end{array}$ & $\mathrm{X}$ & $\sqrt{ }$ & $\mathrm{X}$ & & $\sqrt{ }$ & $\mathrm{X}$ & $\sqrt{ }$ & $\sqrt{ }$ \\
\hline $\begin{array}{l}\text { Car park space } \\
\text { for entry and } \\
\text { exit } \\
5400 \mathrm{~mm} \times \\
3600 \mathrm{~m} \\
(\mathrm{~L} \times \mathrm{W})\end{array}$ & $\begin{array}{c}5030 \mathrm{~mm} x \\
2050 \mathrm{~mm} \\
(\mathrm{~L} \times \mathrm{W})\end{array}$ & $\begin{array}{c}6220 \mathrm{~mm} x \\
2440 \mathrm{~mm} \\
(\mathrm{~L} \times \mathrm{W})\end{array}$ & $\begin{array}{l}3900 \mathrm{~mm} x \\
2260 \mathrm{~mm} \\
(\mathrm{Lx} \mathrm{W})\end{array}$ & & $\begin{array}{c}\text { Parking A and B : } \\
\text { 6500mm x 2170mm (L } \\
\text { x W) } \\
\text { Parking C : } 5000 \mathrm{~mm} \mathrm{x} \\
\text { 2400mm }(\mathrm{L} \times \mathrm{W})\end{array}$ & $\begin{array}{c}2700 \mathrm{~mm} x \\
4800 \mathrm{~mm}(\mathrm{~L} \mathrm{x} \mathrm{W})\end{array}$ & $\begin{array}{c}5670 \mathrm{~mm} x \\
2540 \mathrm{~mm} \\
(\mathrm{~L} \mathrm{x} \mathrm{W})\end{array}$ & $\begin{array}{c}4750 \mathrm{~mm} x \\
2930 \mathrm{~mm}(\mathrm{~L} \mathrm{x} \mathrm{W})\end{array}$ \\
\hline $\begin{array}{l}\text { Transfer area } \\
\text { minimum of } \\
1200 \mathrm{~m}\end{array}$ & $X$ & $\mathrm{X}$ & $\mathrm{X}$ & & $\mathrm{X}$ & $\mathrm{X}$ & $\mathrm{X}$ & $\mathrm{X}$ \\
\hline
\end{tabular}


International Journal of Recent Technology and Engineering (IJRTE) ISSN: 2277-3878, Volume-8 Issue-2S10, September 2019

\begin{tabular}{|c|c|c|c|c|c|c|c|}
\hline $\begin{array}{l}\text { Signage height } \\
\text { from floor } \\
\text { surface: } \\
1500 \mathrm{~m}\end{array}$ & $\begin{array}{c}\text { Signage height } \\
\text { 1900m }\end{array}$ & & $\begin{array}{c}\text { Signage } \\
\text { height } \\
900 \mathrm{~mm}\end{array}$ & $\begin{array}{c}\text { Signage height } \\
1600 \mathrm{~mm}\end{array}$ & $\begin{array}{c}\text { Signage height } \\
1600 \mathrm{~mm}\end{array}$ & & $X$ \\
\hline $\begin{array}{l}\text { Separate lift to } \\
\text { access the } \\
\text { building }\end{array}$ & $\sqrt{ }$ & & & $X$ & $X$ & & \\
\hline $\begin{array}{l}\text { Minimum } \\
\text { height of } \\
\text { entrance to } \\
\text { parking area: } \\
\text { 2400mm }\end{array}$ & $\begin{array}{c}\text { Height } \\
\text { 2400mm }\end{array}$ & & $\sqrt{ }$ & & & & \\
\hline $\begin{array}{l}\text { Disabled } \\
\text { parking is } \\
\text { provided near } \\
\text { the main } \\
\text { entrance and is } \\
\text { not more than } \\
50 \mathrm{~m} \text { away. }\end{array}$ & & $\sqrt{ }$ & $\sqrt{ }$ & $\sqrt{ }$ & $\begin{array}{l}\text { More than } 50 \mathrm{~m} \\
\text { from main } \\
\text { entrance }\end{array}$ & $\sqrt{ }$ & $\sqrt{ }$ \\
\hline
\end{tabular}

Table 1: Disabled parking facilities at UKM

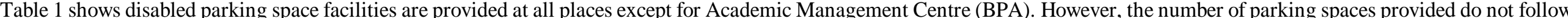

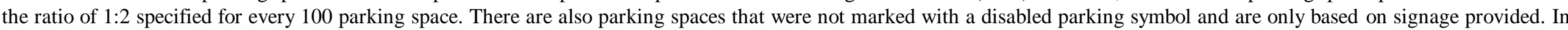

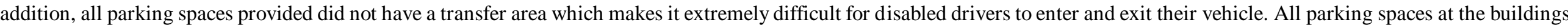

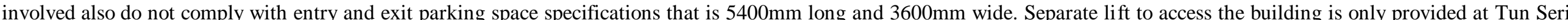
Lanang Library (PTSL) 
B. STAIRS

\begin{tabular}{|c|c|c|c|c|c|c|c|c|}
\hline $\begin{array}{l}\text { Universal } \\
\text { design indoor } \\
\text { criteria }\end{array}$ & $\begin{array}{l}\text { Perpustakaan } \\
\text { Tun Seri } \\
\text { Lanang } \\
\text { (PTSL) }\end{array}$ & PUSANIKA & UNIKEB & $\begin{array}{c}\text { ACADEMIC } \\
\text { MANAGEMENT } \\
\text { SECTION } \\
\text { (BPA) }\end{array}$ & $\begin{array}{c}\text { FACULTY OF } \\
\text { SOCIAL SCIENCES } \\
\text { AND HUMANITIES }\end{array}$ & $\begin{array}{l}\text { FACULTY OF } \\
\text { EDUCATION }\end{array}$ & $\begin{array}{c}\text { FACULTY OF } \\
\text { ECONOMICS } \\
\text { AND } \\
\text { MANAGEMENT }\end{array}$ & $\begin{array}{l}\text { FACULTY OF } \\
\text { SCIENCE AND } \\
\text { TECHNOLOGY }\end{array}$ \\
\hline $\begin{array}{l}\text { Step height of } \\
\text { not more than } \\
180 \mathrm{~mm} \text { and } \\
\text { length of not } \\
\text { less than } \\
260 \mathrm{~mm}\end{array}$ & $\begin{array}{l}\text { Height } \\
140 \mathrm{~mm} \\
\text { Length } \\
280 \mathrm{~mm}\end{array}$ & $\begin{array}{l}\text { Height } \\
140 \mathrm{~mm} \\
\text { Length } \\
270 \mathrm{~mm}\end{array}$ & $\begin{array}{l}\text { Height } \\
152.4 \mathrm{~mm} \\
\text { Length } \\
254 \mathrm{~mm}\end{array}$ & $\begin{array}{l}\text { Height } \\
160 \mathrm{~mm} \\
\text { Length } \\
280 \mathrm{~mm}\end{array}$ & $\begin{array}{l}\text { Height } 120 \mathrm{~mm} \\
\text { Length } 190 \mathrm{~mm}\end{array}$ & $\begin{array}{c}\text { Height } \\
150 / 140(\mathrm{~mm}) \\
\text { Length } \\
250 / 280(\mathrm{~mm})\end{array}$ & $\begin{array}{l}\text { Height } 150 \mathrm{~mm} \\
\text { Lenth } 310 \mathrm{~mm}\end{array}$ & $\begin{array}{l}\text { Height } 150 \mathrm{~mm} \\
\text { Length } 260 \mathrm{~mm}\end{array}$ \\
\hline $\begin{array}{l}\text { Landing area } \\
\text { that is free } \\
\text { from barriers, } \\
\text { width is not } \\
\text { less than } \\
1500 \mathrm{~mm}\end{array}$ & $\begin{array}{l}\text { Landing area } \\
\text { width } \\
2940 \mathrm{~mm}\end{array}$ & Very spacious & $\begin{array}{l}\text { Landing } \\
\text { area width } \\
3400 \mathrm{~mm}\end{array}$ & $\begin{array}{l}\text { Landing area width } \\
2880 \mathrm{~mm}\end{array}$ & $\begin{array}{c}\text { Landing area width } \\
1200 \mathrm{~mm}\end{array}$ & $\begin{array}{c}\text { Landing area } \\
\text { width } \\
1460 \mathrm{~mm} / 1800 \\
\mathrm{~mm}\end{array}$ & $\begin{array}{l}\text { Landing area width } \\
1750 \mathrm{~mm}\end{array}$ & $\begin{array}{l}\text { Landing area } \\
\text { width } 2000 \mathrm{~mm}\end{array}$ \\
\hline $\begin{array}{l}\text { The } 300 \mathrm{~mm} \\
\text { wide warning } \\
\text { type tactile is } \\
\text { mounted at a } \\
\text { distance of } \\
300 \mathrm{~mm} \\
\text { between the } \\
\text { tactile and the } \\
\text { end of the first } \\
\text { step }\end{array}$ & $\mathrm{X}$ & $\mathrm{X}$ & $X$ & $\mathrm{X}$ & $\mathrm{X}$ & $X$ & $\mathrm{X}$ & $X$ \\
\hline
\end{tabular}

Table 3: Stairs facilities at UKM

All indoor stairs that were studied have a height of no more than $180 \mathrm{~mm}$. The width of the landing area also does not exceed $1500 \mathrm{~mm}$ and there is adequate space to accommodate the movements of PWD. However, all of the buildings did not install warning tactile for the visually impaired.

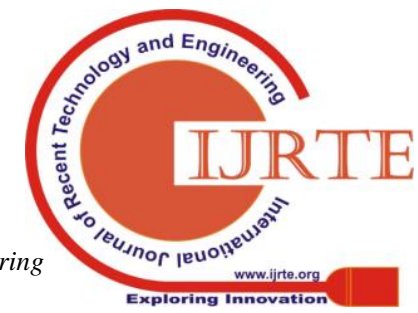


C. LIFTS

\begin{tabular}{|c|c|c|c|c|c|c|c|c|}
\hline $\begin{array}{c}\text { Universal } \\
\text { design indoor } \\
\text { criteria }\end{array}$ & $\begin{array}{l}\text { Perpustakaan } \\
\text { Tun Seri } \\
\text { Lanang } \\
\text { (PTSL) }\end{array}$ & PUSANIKA & UNIKEB & $\begin{array}{c}\text { ACADEMIC } \\
\text { MANAGEMENT } \\
\text { SECTION } \\
\text { (BPA) }\end{array}$ & $\begin{array}{c}\text { FACULTY OF } \\
\text { SOCIAL SCIENCES } \\
\text { AND HUMANITIES }\end{array}$ & $\begin{array}{l}\text { FACULTY OF } \\
\text { EDUCATION }\end{array}$ & $\begin{array}{c}\text { FACULTY OF } \\
\text { ECONOMICS } \\
\text { AND } \\
\text { MANAGEMENT }\end{array}$ & $\begin{array}{c}\text { FACULTY OF } \\
\text { SCIENCE AND } \\
\text { TECHNOLOGY }\end{array}$ \\
\hline $\begin{array}{l}\text { Interior size of } \\
\text { lift : not less } \\
\text { than }(1100 \mathrm{~mm} \\
\mathrm{x} 1400 \mathrm{~mm})\end{array}$ & $\begin{array}{c}\text { PWD lift } \\
\text { interior size }\end{array}$ & NO LIFT & $\begin{array}{l}\mathrm{L}: 1050 \mathrm{~mm} \\
\mathrm{X} \\
\mathrm{W}: 1200 \mathrm{~mm}\end{array}$ & $\begin{array}{c}\mathrm{L}: 1250 \mathrm{~mm} \mathrm{x} \\
\mathrm{W}: 1600\end{array}$ & $\begin{array}{l}\mathrm{L}: 1600 \mathrm{~mm} \mathrm{x} \\
\mathrm{W}: 1700 \mathrm{~mm}\end{array}$ & NO LIFT & $\begin{array}{l}\text { PWD lift interior } \\
\text { size }\end{array}$ & $\begin{array}{l}\text { PWD lift interior } \\
\text { size }\end{array}$ \\
\hline $\begin{array}{l}\text { Mirror is } \\
\text { installed } \\
\text { opposite the lift } \\
\text { door }\end{array}$ & $\begin{array}{c}\text { L: } 1630 \mathrm{~mm} x \\
\mathrm{~W}: 1730 \mathrm{~mm} \text {, } \\
\text { mirror is } \\
\text { installed }\end{array}$ & & X & X & X & & 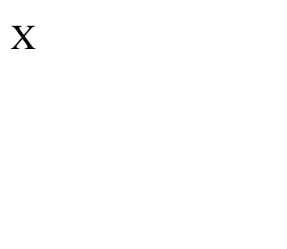 & X \\
\hline $\begin{array}{l}\text { Interior size of } \\
\text { lift trolley and } \\
\text { stretcher is not } \\
\text { less than } \\
(1200 \mathrm{~mm} x \\
2300 \mathrm{~mm})\end{array}$ & $\begin{array}{l}\mathrm{L}: 1640 \mathrm{~mm} \mathrm{x} \\
\mathrm{W}: 1890 \mathrm{~mm}\end{array}$ & & $\begin{array}{l}\mathrm{L}: 1050 \mathrm{~mm} \\
\mathrm{x} \\
\mathrm{W}: 1200 \mathrm{~mm}\end{array}$ & $\begin{array}{l}\mathrm{L}: 1250 \mathrm{~mm} \times \\
\mathrm{W}: 1600 \mathrm{~mm}\end{array}$ & $\mathrm{X}$ & & $X$ & $\begin{array}{l}\mathrm{L}: 1400 \mathrm{~mm} x \\
\mathrm{~W}: 1960 \mathrm{~mm}\end{array}$ \\
\hline $\begin{array}{l}\text { Door width is } \\
\text { not less than } \\
900 \mathrm{~mm} \text {. }\end{array}$ & $\begin{array}{c}\text { Door width } \\
1100 \mathrm{~mm}\end{array}$ & & $\begin{array}{l}\text { Door width } \\
900 \mathrm{~mm}\end{array}$ & $\begin{array}{l}\text { Door width } \\
1100 \mathrm{~mm}\end{array}$ & $\begin{array}{l}\text { Door width } \\
1100 \mathrm{~mm}\end{array}$ & & $\begin{array}{l}\text { Door width } \\
1120 \mathrm{~mm}\end{array}$ & $\begin{array}{l}\text { Door width } \\
1060 \mathrm{~mm}\end{array}$ \\
\hline
\end{tabular}


Accessibility Assessment of Facilities for Persons with Disabilities at Public Institutes of Higher Education

\begin{tabular}{|c|c|c|c|c|c|c|}
\hline $\begin{array}{l}\text { Minimum of } 1 \\
\text { handrail is } \\
\text { provided inside } \\
\text { the lift : } \\
\text { i. Measures } \\
\text { between } 40 \\
\mathrm{~mm}-50 \mathrm{~mm} \\
\text { ii. Handrail } \\
\text { height of } \\
(800 \mathrm{~mm}-900 \\
\mathrm{mm}) \text { from the } \\
\text { floor }\end{array}$ & $X$ & $X$ & $\sqrt{ }$ & $X$ & $X$ & $X$ \\
\hline $\begin{array}{l}\text { Braille } \\
\text { lettering is } \\
\text { provided on the } \\
\text { lift control } \\
\text { buttons and is } \\
\text { easily } \\
\text { identifiable. }\end{array}$ & $X$ & $X$ & $\sqrt{ }$ & $X$ & $X$ & $X$ \\
\hline $\begin{array}{l}\text { Clear and } \\
\text { easily } \\
\text { identifiable } \\
\text { emergency } \\
\text { intercom } \\
\text { button. }\end{array}$ & $\sqrt{ }$ & $\sqrt{ }$ & $\sqrt{ }$ & $\sqrt{ }$ & $\sqrt{ }$ & \\
\hline $\begin{array}{l}\text { Sound system } \\
\text { alert for each } \\
\text { level. }\end{array}$ & $X$ & $X$ & $\sqrt{ }$ & $\mathrm{X}$ & $\mathrm{X}$ & $\mathrm{X}$ \\
\hline
\end{tabular}


International Journal of Recent Technology and Engineering (IJRTE) ISSN: 2277-3878, Volume-8 Issue-2S10, September 2019

\begin{tabular}{|c|c|c|c|c|c|c|}
\hline $\begin{array}{l}\text { Lift control } \\
\text { buttons panel } \\
\text { are installed } \\
\text { between } \\
900 \mathrm{~mm}- \\
1200 \mathrm{~mm} \text { from } \\
\text { floor surface } \\
\text { inside and } \\
\text { outside the lift. }\end{array}$ & $\begin{array}{l}\text { Outside lift: } \\
920 \mathrm{~mm} \\
\text { Inside lift: } \\
1100 \mathrm{~mm}\end{array}$ & $\begin{array}{l}\text { Outside lift: } \\
\text { 1000mm } \\
\text { Inside lift : } \\
1100 \mathrm{~mm}\end{array}$ & $\begin{array}{c}\text { Outside lift : } \\
1100 \mathrm{~mm} \\
\text { Inside lift: } 840 \mathrm{~mm}\end{array}$ & $\begin{array}{l}\text { Outside lift: } 900 \mathrm{~mm} \\
\text { Inside lif: } 1000 \mathrm{~mm}\end{array}$ & $\begin{array}{l}\text { Lift control buttons } \\
\text { panel } 1120 \mathrm{~mm}\end{array}$ & $\begin{array}{c}\text { Lift control } \\
\text { buttons panel } \\
1150 \mathrm{~mm}\end{array}$ \\
\hline
\end{tabular}

Table 4: Lift facilities at UKM

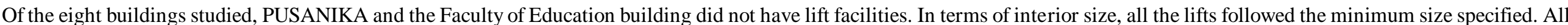

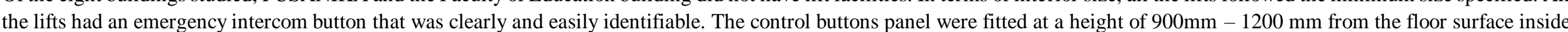

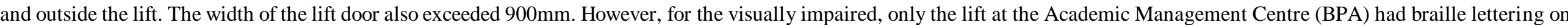
the lift control button, sound system alerts for every level and handrails inside the lift

\section{TOILETS}

\begin{tabular}{|c|c|c|c|c|c|c|c|c|}
\hline $\begin{array}{c}\text { Universal } \\
\text { design indoor } \\
\text { criteria }\end{array}$ & $\begin{array}{l}\text { Perpustakaan } \\
\text { Tun Seri } \\
\text { Lanang } \\
\text { (PTSL) }\end{array}$ & PUSANIKA & UNIKEB & $\begin{array}{c}\text { ACADEMIC } \\
\text { MANAGEMENT } \\
\text { SECTION } \\
\text { (BPA) }\end{array}$ & $\begin{array}{c}\text { FACULTY OF } \\
\text { SOCIAL SCIENCES } \\
\text { AND HUMANITIES }\end{array}$ & $\begin{array}{l}\text { FACULTY OF } \\
\text { EDUCATION }\end{array}$ & $\begin{array}{c}\text { FACULTY OF } \\
\text { ECONOMICS } \\
\text { AND } \\
\text { MANAGEMENT }\end{array}$ & $\begin{array}{c}\text { FACULTY OF } \\
\text { SCIENCE AND } \\
\text { TECHNOLOGY }\end{array}$ \\
\hline
\end{tabular}


Accessibility Assessment of Facilities for Persons with Disabilities at Public Institutes of Higher Education

\begin{tabular}{|c|c|c|c|c|c|c|c|c|}
\hline $\begin{array}{l}\text { Provide PWD } \\
\text { needs such as: } \\
\text { i. At least one } \\
\text { toilet cubicle } \\
\text { that can be } \\
\text { accessed by a } \\
\text { wheelchair } \\
\text { ii. The toilet } \\
\text { that can be } \\
\text { accessed by a } \\
\text { wheelchair } \\
\text { must have a } \\
\text { sink. }\end{array}$ & $\begin{array}{c}\text { One disabled } \\
\text { toilet on level } 4 \\
\qquad \sqrt{ }\end{array}$ & $\begin{array}{l}\text { NO DISABLED } \\
\text { TOILET }\end{array}$ & $\begin{array}{c}\text { NO } \\
\text { DISABLED } \\
\text { TOILET }\end{array}$ & $\begin{array}{l}\text { One disabled toilet } \\
\text { on level } 1 \\
\sqrt{ }\end{array}$ & $\begin{array}{c}\text { Three disabled toilets on } \\
\text { level } 3 \text { and } 4 \\
\text { Only one toilet has a } \\
\text { sink }\end{array}$ & $\begin{array}{c}\text { Five disabled } \\
\text { toilets are } \\
\text { provided on } \\
\text { level } 1 \text { and } 2 \\
\\
\text { All toilets have a } \\
\text { sink }\end{array}$ & $\begin{array}{l}\text { Two disabled } \\
\text { toilets at every end } \\
\text { of the building } \\
\sqrt{ }\end{array}$ & $\begin{array}{l}\text { Two disabled } \\
\text { toilets }\end{array}$ \\
\hline $\begin{array}{l}\text { Symbols are } \\
\text { easily } \\
\text { identifiable } \\
\text { and is } \\
\text { displayed for } \\
\text { the use of the } \\
\text { visually } \\
\text { impaired and } \\
\text { mentally } \\
\text { impaired. }\end{array}$ & $\begin{array}{c}\text { Disabled } \\
\text { symbol only } \\
\text { displayed on } \\
\text { the toilet door }\end{array}$ & & & $\begin{array}{l}\text { Disabled symbol } \\
\text { only displayed on } \\
\text { the toilet door }\end{array}$ & $\begin{array}{l}\text { Disabled symbol only } \\
\text { displayed on the toilet } \\
\text { door }\end{array}$ & $\begin{array}{l}\text { Disabled symbol } \\
\text { displayed on the } \\
\text { front of the toilet } \\
\text { door }\end{array}$ & $\begin{array}{l}\text { Disabled symbol } \\
\text { displayed on the } \\
\text { front of the toilet } \\
\text { door }\end{array}$ & $\begin{array}{l}\text { Disabled symbol } \\
\text { displayed on the } \\
\text { front of the toilet } \\
\text { door. }\end{array}$ \\
\hline $\begin{array}{l}\text { Minimum } \\
\text { entrance of } \\
900 \mathrm{~mm} \mathrm{x} \\
900 \mathrm{~mm} .\end{array}$ & $\begin{array}{l}\mathrm{L}: 810 \mathrm{~mm} \\
\mathrm{~W}: 810 \mathrm{~mm}\end{array}$ & & & $\begin{array}{l}\mathrm{L}: 900 \mathrm{~mm} \\
\mathrm{~W}: 950 \mathrm{~mm}\end{array}$ & $\begin{array}{l}\mathrm{L}: 920 / 1000 \mathrm{~mm} \\
\mathrm{~W}: 900 / 950 \mathrm{~mm}\end{array}$ & $\begin{array}{l}\mathrm{L}: 920 \mathrm{~mm} \\
\mathrm{~W}: 900 \mathrm{~mm}\end{array}$ & $\begin{array}{l}\mathrm{L}: 1580 \mathrm{~mm} \\
\mathrm{~W}: 1580 \mathrm{~mm}\end{array}$ & $1000 \mathrm{~mm}$ \\
\hline
\end{tabular}


International Journal of Recent Technology and Engineering (IJRTE) ISSN: 2277-3878, Volume-8 Issue-2S10, September 2019

\begin{tabular}{|c|c|c|c|c|c|c|}
\hline $\begin{array}{l}\text { Has a } \\
\text { minimum } \\
\text { space of } \\
1700 \mathrm{~mm} \mathrm{x} \\
2200 \mathrm{~mm}(\mathrm{~L} \mathrm{x} \\
\mathrm{W}) \\
\text { The toilet is not } \\
\text { slippery }\end{array}$ & $\begin{array}{c}\mathrm{L}: 1860 \mathrm{~mm} \\
\mathrm{~W}: 1660 \mathrm{~mm} \\
\\
\sqrt{ }\end{array}$ & $\begin{array}{c}\mathrm{L}: 1700 \mathrm{~mm} \\
\mathrm{~W}: 1400 \mathrm{~mm} \\
\\
\sqrt{ }\end{array}$ & $\sqrt{ }$ & $\sqrt{ }$ & $\begin{array}{c}\text { L: } 1700 \mathrm{~mm} \\
\mathrm{~W}: 2190 \mathrm{~mm} \\
\\
\mathrm{X}\end{array}$ & $\begin{array}{c}\mathrm{L}: 1800 \mathrm{~mm} \\
\mathrm{W:} 1830 \mathrm{~mm} \\
\\
\sqrt{ }\end{array}$ \\
\hline $\begin{array}{l}\text { Toilet door } \\
\text { opens } \\
\text { outwards, has a } \\
\text { minimum } \\
\text { opening of } 800 \\
\text { mm. It is } \\
\text { recommended } \\
\text { to use a sliding } \\
\text { door }\end{array}$ & $\begin{array}{l}\text { Uses a sliding } \\
\text { door }\end{array}$ & $\begin{array}{c}\text { Door opens by } \\
\text { pushing inwards }\end{array}$ & $\begin{array}{l}\text { First design: A normal } \\
\text { door that opens from the } \\
\text { inside. Door opening is } \\
\text { 850mm } \\
\text { Second design: Uses a } \\
\text { sliding door. Door } \\
\text { opening of } 900 \mathrm{~mm}\end{array}$ & $\begin{array}{c}\text { Door opens by } \\
\text { pushing inwards }\end{array}$ & Uses a sliding door & $\begin{array}{c}\text { Door opens by } \\
\text { pushing inwards }\end{array}$ \\
\hline
\end{tabular}


Accessibility Assessment of Facilities for Persons with Disabilities at Public Institutes of Higher Education

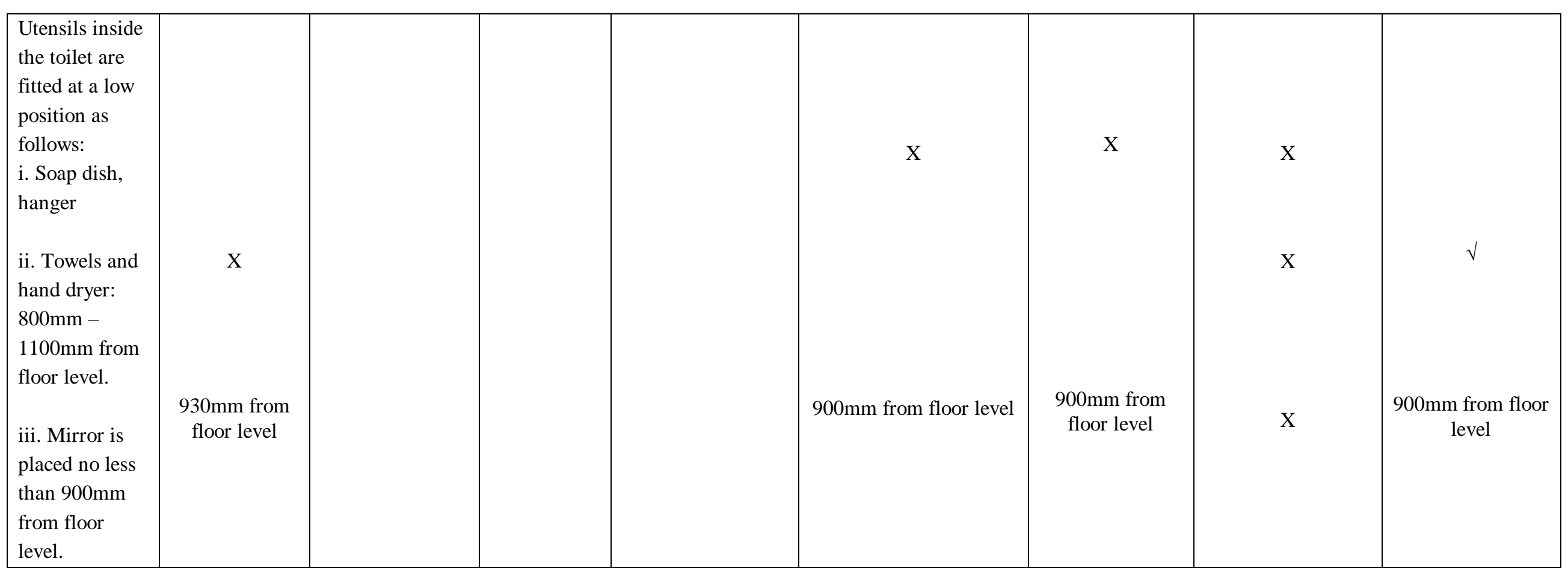


International Journal of Recent Technology and Engineering (IJRTE) ISSN: 2277-3878, Volume-8 Issue-2S10, September 2019

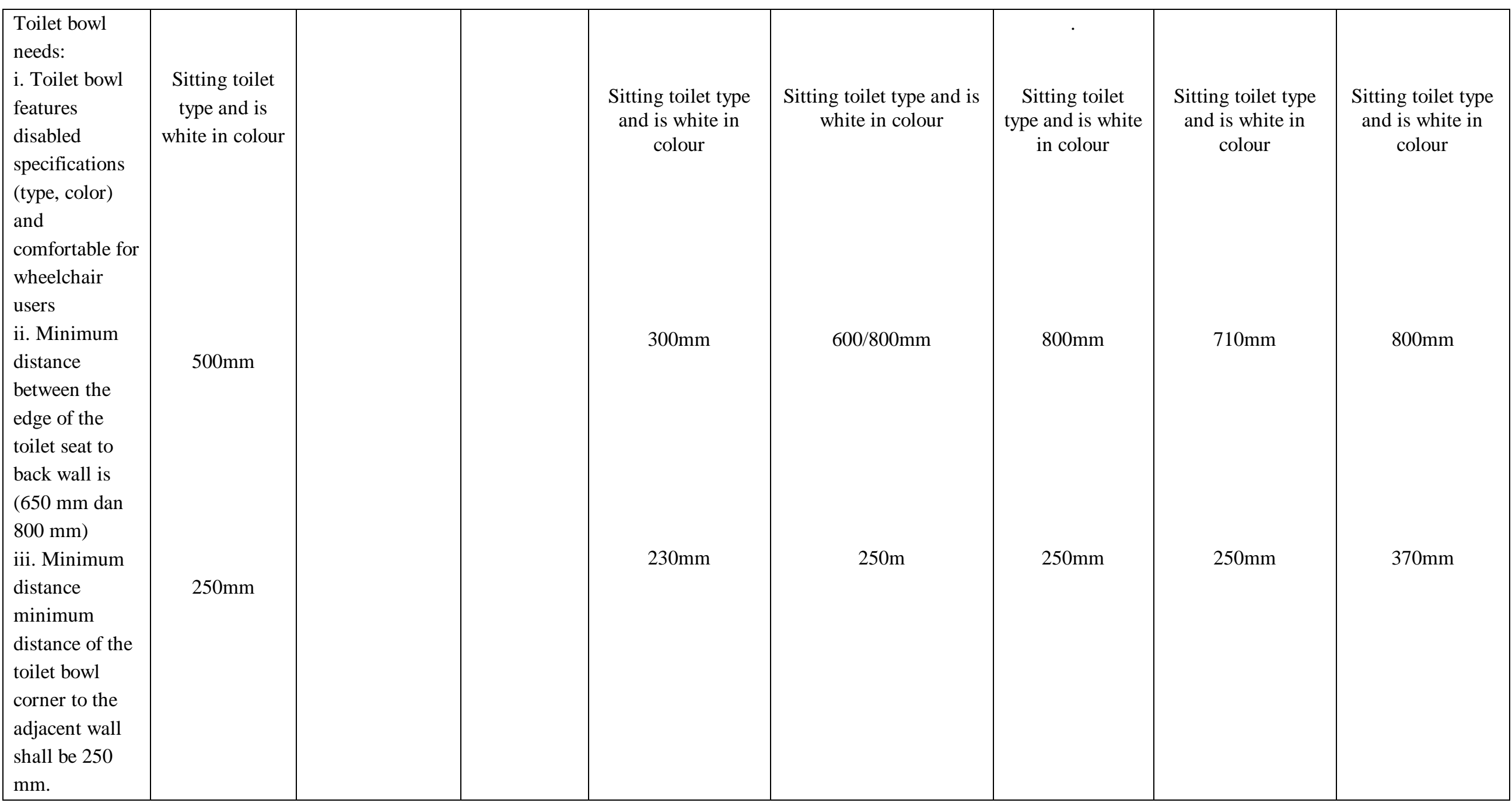


Accessibility Assessment of Facilities for Persons with Disabilities at Public Institutes of Higher Education

\begin{tabular}{|c|c|c|c|c|c|c|}
\hline $\begin{array}{l}\text { Grab rails on } \\
\text { both sides } \\
\text { (either } \\
\text { drop-down or } \\
\text { permanently } \\
\text { fixed to the } \\
\text { wall) is } \\
\text { provided at a } \\
\text { distance of } 300 \\
\text { mm to } 350 \mathrm{~mm} \\
\text { from toilet } \\
\text { bowl. }\end{array}$ & $\begin{array}{l}\text { Only one grab } \\
\text { rail is provided } \\
\text { next to the } \\
\text { toilet bowl }\end{array}$ & $\begin{array}{l}\text { There are no grab } \\
\text { rails provided next } \\
\text { to the toilet bowl }\end{array}$ & $\begin{array}{l}\text { Both toilets have one } \\
\text { grab rail next to the } \\
\text { toilet bowl }\end{array}$ & $\begin{array}{l}\text { All toilets have } \\
\text { only one grab } \\
\text { rail }\end{array}$ & $\begin{array}{l}\text { Only one grab rail } \\
\text { is provided next to } \\
\text { the toilet bowl }\end{array}$ & $\begin{array}{l}\text { Only one grab rail } \\
\text { is provided next to } \\
\text { the toilet bowl }\end{array}$ \\
\hline
\end{tabular}

\section{Table 5: Disabled toilet facilities at UKM}

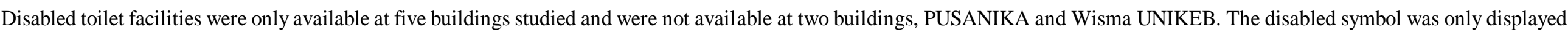

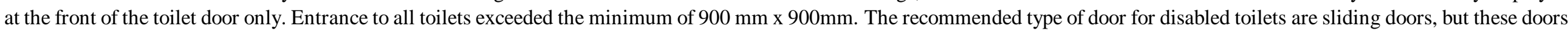

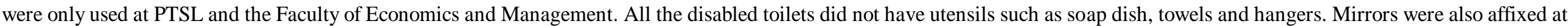

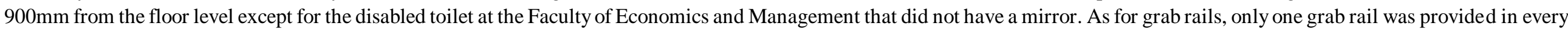
toilet.

\section{E. PATHWAYS OR RAMPS}

\begin{tabular}{|c|c|c|c|c|c|c|c|c|}
\hline $\begin{array}{l}\text { Universal } \\
\text { design indoor } \\
\text { criteria }\end{array}$ & $\begin{array}{l}\text { Perpustakaan } \\
\text { Tun Seri } \\
\text { Lanang } \\
\text { (PTSL) }\end{array}$ & PUSANIKA & UNIKEB & $\begin{array}{c}\text { ACADEMIC } \\
\text { MANAGEMENT } \\
\text { SECTION } \\
\text { (BPA) }\end{array}$ & $\begin{array}{c}\text { FACULTY OF } \\
\text { SOCIAL SCIENCES } \\
\text { AND HUMANITIES }\end{array}$ & $\begin{array}{l}\text { FACULTY OF } \\
\text { EDUCATION }\end{array}$ & $\begin{array}{c}\text { FACULTY OF } \\
\text { ECONOMICS } \\
\text { AND } \\
\text { MANAGEMEN } \\
\text { T }\end{array}$ & $\begin{array}{c}\text { FACULTY OF } \\
\text { SCIENCE AND } \\
\text { TECHNOLOGY }\end{array}$ \\
\hline
\end{tabular}


International Journal of Recent Technology and Engineering (IJRTE) ISSN: 2277-3878, Volume-8 Issue-2S10, September 2019

\begin{tabular}{|c|c|c|c|c|c|c|c|c|}
\hline $\begin{array}{l}\text { Provided for any } \\
\text { changes in } \\
\text { surface height. } \\
\text { Stairs are } \\
\text { provided } \\
\text { adjacent to the } \\
\text { ramp if the level } \\
\text { difference } \\
\text { exceeds } \\
300 \mathrm{~mm} .\end{array}$ & $\sqrt{ }$ & $\sqrt{ }$ & $\sqrt{ }$ & NO RAMP & $\sqrt{ }$ & $\sqrt{ }$ & $\sqrt{ }$ & $\sqrt{ }$ \\
\hline $\begin{array}{l}\text { Ramp width is } \\
\text { not less than } \\
1200 \mathrm{~mm} .\end{array}$ & $\begin{array}{c}\text { Width: } \\
\text { 1260mm but is } \\
\text { obstructed by a } \\
\text { pole }\end{array}$ & $\begin{array}{l}\text { Width : } \\
1250 \mathrm{~mm}\end{array}$ & $\begin{array}{l}\text { Width : } \\
\text { 1350mm }\end{array}$ & & $\begin{array}{l}\text { Ramp A :1400mm } \\
\text { Ramp B : } 1000 \mathrm{~mm}\end{array}$ & $\begin{array}{l}\text { Width: } \\
\text { 1300mm }\end{array}$ & $\begin{array}{c}\text { Width: } 1525 \mathrm{~mm} \\
\text { Handrails } \\
1000 \mathrm{~mm}\end{array}$ & Width: $1510 \mathrm{~mm}$ \\
\hline $\begin{array}{l}\text { Handrails are } \\
\text { provided on } \\
\text { both sides of } \\
\text { ramps that are } \\
\text { longer than } \\
800 \mathrm{~mm} \text {. }\end{array}$ & $\sqrt{ }$ & $\mathrm{X}$ & $\begin{array}{l}\text { Handrail is } \\
\text { provided } \\
\text { only on the } \\
\text { left side }\end{array}$ & & $X$ & $\sqrt{ }$ & Only on one side & $\sqrt{ }$ \\
\hline $\begin{array}{l}\text { Stable ramp } \\
\text { surface and is } \\
\text { not slippery in } \\
\text { both dry and wet } \\
\text { conditions. }\end{array}$ & $\mathrm{X}$ & $\sqrt{ }$ & $\sqrt{ }$ & & $\sqrt{ }$ & $\sqrt{ }$ & $\sqrt{ }$ & $\sqrt{ }$ \\
\hline
\end{tabular}

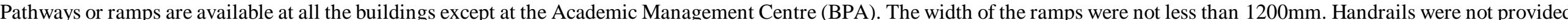

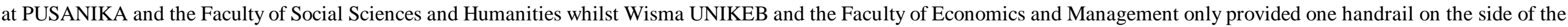
ramp. The ramp surface at PUSANIKA is unstable and slippery as compared to the ramps at the other buildings that were stable and not slippery.

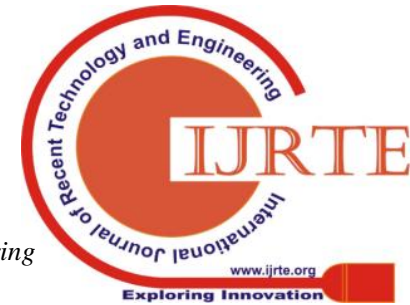




\section{DISCUSSION}

Disabled facilities provided at a place should include the input and consent of the disabled to ensure the facilities meet their needs. Bodaghi and Zainab (2012) in their study found that there are differences of opinion between architects and disabled users regarding the facilities provided, especially the pathways or ramps and in-house layout of the library where their study was conducted. In the opinion of architects, the facilities provided are in good condition, but for the disabled user, the facilities provided are inadequate. In this case, there is a need for mutual understanding between the facility provider and user to ensure that the facilities provided are easily accessible as well as comply with the structural specifications that have been set. At PTSL UKM (the library), only one disabled parking was provided, even though the area is a focal point for students. Similarly, toilets for the disabled are only available on level 4 of the building. It is common knowledge that individuals with physical and visual disabilities face difficulties in moving. Thus, ideally the number of both facilities should be increased and provided at a building level that can be easily accessed by the disabled.

From the observations made, the signage and direction of the facilities for the disabled are still lacking. For example, at the toilets. Disabled toilet signage are only stuck on the front of the toilet door. This will confuse disabled users whether the facility is available or not at the area. The researchers recommends that specific signage and directions to disabled facilities are provided at the foyer and parking area of every faculty building and other resource centres to facilitate the movement of PWD at the buildings.

The efforts already carried out to provide disabled facilities are commendable. However, to what extent are these facilities fully accessible to disabled users? As an example, disabled toilets. Most of the doors at disabled toilets open inwards or outwards which will certainly make it difficult for the disabled using wheelchairs. Lifts provided also lack a sound system that announces their level. This makes it difficult for the visually impaired to use lifts on their own and if there is an absence of braille lettering on the control buttons, it will be even more difficult. Facility providers should provide comprehensive facilities starting from the construction phase up to ensuring the accessibility of the facilities for PWD. The findings of the study is seen as a guide to the management of UKM to provide easily accessible facilities to the disabled based on the criteria set out in a universal design building. More attention to disabled facilities in UKM is needed as the UKM Bangi campus is made up of older buildings that require a lot of renovation to improve the facilities for the disabled.

A study was carried out to identify the factors that influence the improvement of disabled facilities at hospitals [15]. These factors appear to be applicable in other places as well. Among the factors are: i) regular maintenance to ensure the facilities are functioning properly, ii) provision of disabled facilities beginning from the construction phase of the building, iii) the role of the government in ensuring an area is PWD friendly and is fully accessible, iv) more barrier-free facilities in public places to avoid the constraints faced by the disabled as well as, v) the role and perception of society to cooperate in ensuring that the facilities provided are always in good condition.

The awareness of equality rights for PWD especially in terms of access to the physical surroundings should be nurtured from the beginning in particular among professional practitioners involved in the construction sector such as architects and contractors. This is because they are the individuals responsible for the construction of a building. Realizing this, Universiti Islam Antarabangsa Malaysia (UIAM) has created an elective subject, 'Barrier Free Architecture' that can be taken by architecture students at the university. This subject is seen to provide insight to future architecture graduates on the concept of barrier-free, design for all, universal design and inclusive design [16]. Such subjects should be introduced in every institute of higher learning to expose all students to the needs of PWD.

In addition, the government, especially the municipal councils, should take the initiative of increasing the number of staff responsible for approving and supervising the construction of a building. This is because there is still a shortage of staff with expertise in the universal design code of practice [17].

\section{CONCLUSION}

In conclusion, it can be seen that there are basic facilities needed by PWD at several faculties and student resource centres in UKM. However, these facilities are not enough and do not fulfil all the specifications outlined by universal design criteria. Therefore, the university is recommended to formulate an action plan that is more comprehensive and introduce an inclusive policy for PWD to preserve the rights and needs of the disabled. Everybody including management, students, educators and staff at public institutes of higher learning need to be more aware of the needs of the disabled and strive to fulfil their rights in the same way as others. In addition, maintenance of existing facilities should also be undertaken on a regular basis so that the wellbeing of PWD at the university including students, staff and visitors is completely guaranteed. In short, various parties at all levels of the university should play a proactive role in providing fully accessible facilities for PWD. 


\section{REFERENCES}

1. Bodaghi, N.B. and Zainab, N.A.. Examining the accessibility and facility for the disabled in public and university library buildings in Iran Information Development, 2013, 29(1): 1-10.

2. Hill, J.L., Accessibility: Students with disabilities in universities in Canada Canadian Journal of Higher Education Vol. XXII-1, 1992, 48-82.

3. Hazlin Falina Rosli \& Safura Ahmad Sabri, Halangan Fasiliti Pelajar Orang Kurang Upaya (OKU) Di Institusi Pengajian Tinggi Di Lembah Klang, International Journal for Studies on Children, Women, Elderly And Disabled, 2017, Vol. 2

4. Fatimah Abdullah. 2009. Keperluan Kemudahan untuk Orang Kurang Upaya Kes di Universiti Kebangsaan Malaysia, Persidangan Psikologi Malaysia, 2009.

5. Aizan Sofia Amin \& Jamiah Manap. Geografi, Kemiskinan dan Wanita Kurang Upaya Di Malaysia. Journal of of Society and Space. 2015. 11(7): 82-91

6. Rahim, A.A. \& Abdullah, F., Access audit on universal design: The case of Kota Kinabalu Water Front. The International Journal of Interdisciplinary Social Sciences, 2009, Volume 4.

7. Nur Amirah Abd Samad, Ismail Said, Asiah Abdul Rahim, Planning Accessibility Strategies And Connectivity For Malaysian Urban Built Environment Studies In Health Technology And Informatics, 2018 256:367-377.

8. Ostroff, Elaine. "Universal Design: The New Paradigm." In Universal Design Handbook, edited by Wolfgang F.E. Preiser and Elaine Ostroff: McGraw-Hill Education, 2001.

9. Saito, Yoko. "Awareness of Universal Design among Facility Managers in Japan and the United States." Automation in Construction 15, no. 4 (7/ 2006): 462-78.

10. Syazwani Abdul Kadir \& Mariam Jamaludin (2012)Applicability of Malaysian Standards and Universal Design in Public Buildings in Putrajaya Asian Journal of Environment-Studies (ajE-Bs), 2012, Vol $3(9)$

11. Tinklin, T. \& Hall, J. Getting round obstacle: disabled students' experiences in higher education in Scotland. Studies in Higher Education, 1999. 24(2), 183-194.

12. Mohd Faizul Ismail \& Norizan Abdul Ghani, Sokongan yang Diperlukan Pelajar OKU Cacat Penglihatan di Universiti Awam Malaysia, Proceeding of International Conference of Empowering Islamic Civilization, 2017, ISBN 978-967-0899-70-1

13. Mohd Reduan Bin Buyung, Haryati Binti Shafii. Kolej Kediaman Lestari: Penelitian Kemudahan Golongan Orang Kurang Upaya (OKU). 2015. Seminar Kebangsaan Majlis Dekan-Dekan Pendidikan Universiti Awam 2015

14. Kamarul Azmi Jasmi. Penyelidikan Kualitatif Dalam Sains Sosial. 2012

15. Yuhainis Abdul Talib, Nurul Izzati Abdul Ghani , Kharizam Ismail \& Nor'Aini Salleh, The Provision of the Disabled Facilities in Public Hospitals. 2016. MATEC Web of Conferences 66, 00081

16. Asiah Abdul Rahim, Ismawi Zen, Nur Amirah Abd. Samad \& Che Raiskandar Che Rahim, Universal Design and Accessibility: Towards Sustainable Built Environment in Malaysia: Three Days of Creativity and Diversity. 2014. Volume 35, 299 - 306.

17. Hazreena Hussein, Naziaty Mohd Yaacob. Development of Accessible Design in Malaysia. Procedia - Social and Behavioral Sciences, 2012. Volume 68, pages 121-133

\section{ACKNOWLEDGEMENT}

A token of appreciation to Universiti Kebangsaan Malaysia for grant funding GGPM-2015-030 and KRA-2018-056 and to all those involved directly or indirectly in this study.

\section{AUTHOR PROFILE}

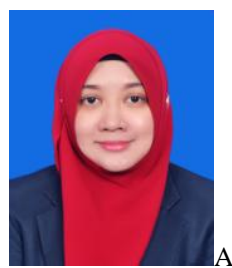

izan Sofia Amin is a senior lecturer at the Faculty of Social Sciences and Humanities, Universiti Kebangsaan Malaysia. She holds a Doctorate of Philosophy specializing in disability studies from the University of Glasgow, United Kingdom. Her areas of expertise are in disability studies, counseling psychology and social work.

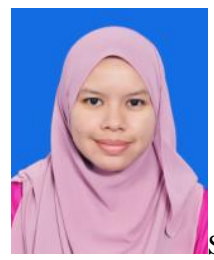
and Organizational Psychology candidate at Universiti Kebangsaan Malaysia. She holds a Bachelor of Psychology (Industry and Organizations) degree from Universiti Kebangsaan Malaysia.

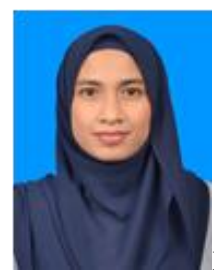

Noremy Md Akhir is a senior lecturer at the Faculty of Social Sciences and Humanities, Universiti Kebangsaan Malaysia. She completed her Master's and Doctor of Philosophy (PhD) in Social Work from University Sains Malaysia. Her research focus is on issues of psychology, crisis intervention and disaster management in the field of social work. 